\section{Cognitive performance during acute alcohol intoxication: The effects of prior task} experience on performance*

\author{
BEN MORGAN JONES \\ Center for Alcohol-Related Studies \\ University of Oklahoma Health Sciences Center, Oklahoma City, Okla. 73190
}

Twenty male medical students were tested on the Shipley-Hartford during an alcohol and placebo condition (counterbalanced) to determine the effects of prior experience on a cognitive task during subsequent performance under alcohol. Significant drug and Drug by Order interaction effects were obtained for the abstraction section where practice effects are typically reported. However, only a significant within-Ss drug effect was obtained for the vocabulary section where practice effects are minimal. A significant correlation was obtained between performance and blood alcohol level only for the group tested first under alcohol. These results indicate that cognitive performance under alcohol may be related to the prior experience that the $S$ has obtained before testing.

Several investigations have reported the effects of alcohol on various cognitive and intellectual tasks (Carpenter, Moore, Snyder, \& Lisansky, 1961; Carpenter \& Ross, 1965; Lewis, Dustman, \& Beck, 1969; Frankenhaeuser, Myresten, \& Jarpe, 1962). Carpenter et al $(1961,1965)$ reported a facilitation of performance on problem-solving and memory tasks at low blood alcohol levels $(0.024 \%$ to $0.055 \%$ ) with deterioration of performance at higher levels $(0.070 \%)$. Lewis et al (1969) failed to find consistent alcohol effects on several cognitive tasks; motor coordination and recitation on a color-naming task were impaired only at a blood alcohol level of $0.06 \%$, while no impairment was found for addition or comparison of spelling of words at $0.03 \%$ or $0.06 \%$. Frankenhaeuser et al (1962) have reported that at blood alcohol levels between $0.08 \%$ and $0.09 \%$, Ss performed significantly more poorly on numerical and spatial tests but not on verbal and inductive tests. The failure of investigators to produce consistent findings concerning the effects of alcohol on cognitive ability may be related to the amount of practice the $S$ receives before he performs the task under alcohol. One method of investigating the contribution of practice to performance is to test Ss under alcohol with and without prior experience with the task. If the same task were administered to $\mathrm{Ss}$ in both an alcohol

*This investigation was supported in part by Public Health Service Grant 14702 from the National Institute of Mental Health subproject "Neuropsychological Concomitants of Alcohol" under the direction of Oscar A. Parsons and Arthur Vega. and placebo condition in a counterbalanced order, it would also be possible to determine whether practice was symmetrical with respect to drug order. That is, does practice during a placebo condition influence subsequent alcohol performance in the same manner that practice under alcohol influences subsequent placebo performance? Another issue is concerned with the relationship between performance and blood alcohol level. Does prior practice on a cognitive task influence the correlation between performance and blood alcohol levels?

In the present investigation, a crossover design was used to evaluate practice effects. This design was chosen since it is often not feasible to train Ss on many cognitive tasks, especially abstracting tasks where a general principle is acquired. This design also provides information concerning possible practice effects that is not available when Ss are trained to maximum performance before administration of alcohol, as in the above-mentioned studies. The Shipley-Hartford was chosen to evaluate practice effects for two reasons. First, it consists of two parts-a vocabulary and abstraction section. There is apparently little practice effect for students on the vocabulary section between first and second testing at 1-week intervals; however, abstraction scores do improve on second testing, indicating some practice effects (Schalock \& Wahler, 1968). Thus it would be possible to evaluate the same Ss on a cognitive test where practice would not be a factor and on one where practice effects would be expected. Therefore, the vocabulary section would serve as a control for the abstraction test. A second reason for using the Shipley-Hartford is that it is quickly administered, requiring a total of $20 \mathrm{~min}$. It is important that testing be carried out rapidly, since the blood alcohol level is continually changing during testing.

Twenty male medical students, ranging in age from 20 to 25 years, served as paid volunteers. Ten Ss were given practice on the Shipley-Hartford under a placebo condition and then tested $48 \mathrm{~h}$ later under alcohol. The other 10 Ss were tested the first day under alcohol (no practice) and then tested in a placebo condition after $48 \mathrm{~h}$.

The Ss were instructed that the purpose of the investigation was to examine the effects of alcohol on behavior during two different occasions. However, each $S$ received alcohol on one occasion and placebo on the other.

A dose of $1.10 \mathrm{ml} / \mathrm{kg}$ of body weight of $95 \%$ USP ethanol mixed with four parts orange drink was administered to all $S s$ in the alcohol condition. This dose was calculated to produce a peak blood alcohol level of $0.08 \%$. Ss in the placebo condition received $4 \mathrm{ml}$ of alcohol floated on top of a comparable volume of orange drink. This was done to insure that these Ss tasted alcohol; however, the dosage was such that its effects would be inconsequential. All Ss were asked to consume the beverage within $15 \mathrm{~min}$. Testing was begun $15 \mathrm{~min}$ after the end of drinking. Each $S$ was administered the Shipley-Hartford vocabulary section first, followed by the abstraction section. The vocabulary section is a 40-item multiple-choice test that has a 10-min time limit. The abstraction section is a 20-item completion test that also has a 10-min time limit. A breath sample (Stephenson Breathalyzer, Model 900) was taken for each $S$ following completion of the abstraction test. The mean blood alcohol level for $20 \mathrm{Ss}$ was $0.078 \%$. Since Ss were tested soon after consuming the beverage, they were still on the ascending limb of the blood alcohol curve.

Analyses consisted of 2 (order) by 2 (drug) factorial analyses of variance with repeated measures on the vocabulary and abstraction portions of the Shipley-Hartford. Simple effects were evaluated by F tests. Correlations were also computed between performance and blood alcohol levels. RESULTS

Means and standard deviations for the Shipley-Hartford vocabulary and abstraction scores are presented in Table 1. A significant drug effect $(\mathrm{F}=6.29, \quad \mathrm{df}=1 / 18, \quad \mathrm{p}<.05)$ was obtained within Ss for the vocabulary 
Table 1

Means and Standard Deviations for Shipley-Hartford Vocabulary and Abstraction Tasks

\begin{tabular}{ccrrrrr} 
& & \multicolumn{2}{c}{ P-A Group } & & \multicolumn{2}{c}{ A-P Group } \\
\cline { 3 - 5 } & & Placebo $_{1}$ & Alcohol $_{2}$ & & Placebo $_{2}$ & Alcohol $_{1}$ \\
\hline Vocabulary & $\mathrm{M}$ & 18.20 & 17.46 & 18.40 & 18.08 \\
Age & $\mathrm{SD}$ & 0.96 & 1.31 & 1.10 & 1.02 \\
Abstraction & $\mathrm{M}$ & 18.42 & 18.74 & 19.70 & 16.64 \\
Age & $\mathrm{SD}$ & 1.42 & 1.50 & 0.84 & 2.24 \\
\hline
\end{tabular}

score, while order and Drug by Order interactions were nonsignificant. A between-groups comparison indicated no significant differences between alcohol and placebo Ss tested on the first day or between the two alcohol or placebo groups. These data indicate that the vocabulary test is sensitive to the effects of alcohol within Ss regardless of the testing order. Since there is no significant practice effect due to prior task experience, it is not surprising that the two alcohol groups do not differ significantly from each other.

For the abstraction task, a significant drug $(F=15.84, \mathrm{df}=1 / 18$, $\mathrm{p}<.01)$ as well as a significant Drug by Order interaction effect $(F=24.10$, df $=1 / 18, \quad p<.01$ ) was obtained. Simple effects indicated that there was a significant alcohol effect only when Ss were tested under alcohol first without prior task experience. That is, a within-Ss improvement was found from alcohol to placebo $(F=39.51$, df $=1 / 18, p<.01$ ) but not from placebo to alcohol $(F=0.43$, df $=1 / 18, \quad p>.10$ ). A between-Ss difference was also found in that $S$ tested under alcohol on the first day performed significantly more poorly than placebo Ss tested on the first day $(F=6.34$, df $=1 / 18, p<.05)$. Most importantly, alcohol Ss tested on the second day after practice on a placebo day performed significantly better than alcohol Ss tested on the first day without practice $(F=8.81$, $\mathrm{df}=1 / 18$, $p<.05$ ) and not significantly different from placebo Ss. This demonstrates that Ss tested in a placebo condition on the abstraction task are able to overcome the effects of alcohol when tested $48 \mathrm{~h}$ later and perform similarly to placebo Ss; however, the reverse does not occur. This demonstrates the asymmetrical properties of practice on this task and may explain the apparently insignificant effect of alcohol on other cognitive tasks where Ss have had extended practice.

Correlations between performance and blood alcohol levels are presented in Table 2. There were no significant correlations between vocabulary scores and blood alcohol levels for either group. However, a significant negative correlation was obtained between abstraction scores and blood alcohol levels for Ss tested the first day under alcohol without prior exposure on the task $(r=-.66, p<.025)$ but not for the alcohol group tested the second day with practice on the task.

Test-retest correlations which were computed for each group to determine the relationship between performance on alcohol and placebo days are also presented in Table 2. High correlations were found for both vocabulary and abstraction scores in the group that received practice in a placebo condition followed by alcohol. However, nonsignificant correlations were obtained in the group that received alcohol on the first day and placebo the second day.

In summary, performance during the alcohol state following a practice period resembled performance in a placebo state. That is, performance was not impaired as compared to placebo testing, blood alcohol level test-retest correlations between placebo and alcohol states were comparable to what would be expected in no-drug states (Ruiz \& Krauss, 1967).

\section{DISCUSSION}

The importance of the practice effect in a placebo state in influencing subsequent performance under alcohol may contribute to the understanding of the inconsistent findings of the influence of alcohol on cognitive ability. Lewis et al (1969) "stabilized learning and practice effects" by administering the cognitive and motor tests six times at weekly intervals before testing under alcohol. They state that "a striking finding of the present study was the resistance of the cognitive and motor task performances to the deleterious effects often attributed to alcohol ingestion." Considering the results of the present investigation, it is likely that the under alcohol contributed significantly to the results. The authors recognized this possibility when they stated that "the extended practice given before administration of alcohol could have stabilized test performance [p. 629]."

However, practice concerning the method of solution may not produce the same type of effects. Carpenter et al $(1961,1965)$, who reported cognitive impairment following alcohol ingestion, demonstrated the was not related to performance, and practice given all $\mathrm{Ss}$ before testing method of problem solving and memory matching but presented new problems during the alcohol condition. Both of these tasks were very complex compared to most cognitive tasks used.

Frankenhaeuser et al (1962) used a design similar to that used in the present investigation and found performance impairment on two of four cognitive tasks. Only eight Ss were used, and no attempt was made to evaluate an order of drug effect. It appears that the two tasks where no alcohol effect was found were very similar to the Shipley-Hartford vocabulary test in that the Ss had to identify a word opposite to the stimulus word (verbal) or identify a four-letter group that did not belong (inductive). It may be that, like the Shipley vocabulary, these tests merely required stored information or identification and no practice effect was present. However, the two tests where a significant difference was found between alcohol and placebo were similar to the Shipley-Hartford abstraction test, since the $S$ had to complete the test actively by multiplying items (multiplication) or counting blocks (spatial). If practice were an important aspect of these two tests, a significant drug effect might be obtained that would be due to the group (four $\mathrm{Ss}$ ) who received the alcohol first and placebo second, with little difference in the other group.

The asymmetrical effects of practice may also partially explain the asymmetrical results of several state-dependent learning studies where dissociation was not found in the placebo-alcohol direction for some tasks (Storm \& Caird, 1967; Goodwin, Powell, Bremer, Hoine, \& Stern, 1969).

Some of the contradictory findings reported concerning the effects of alcohol on cognitive ability may be resolved if the asymmetrical practice effects observed in counterbalanced designs and the potent effects of extended practice in plateau designs are considered.

Table 2

Correlations Between Shipley-Hartford Performance and Blood Alcohol Levels and Test-Retest Correlations

\begin{tabular}{lcc}
\hline $\begin{array}{l}\text { Blood Alcohol } \\
\text { Level and } \\
\text { Performance }\end{array}$ & \multicolumn{2}{c}{ Group } \\
\cline { 2 - 3 } & A-P & P-A \\
\hline 1. Vocabulary & -.23 & +.11 \\
2. Abstraction & $-.66^{*}$ & -.33 \\
Test-Retest & & \\
1. Vocabulary & +.41 & $+.89 \dagger$ \\
2. Abstraction & +.35 & $+.93 \dagger$ \\
\hline * $p .025, f p<.01$ &
\end{tabular}




\section{REFERENCES}

CARPENTER, J., MOORE, O., SNYDER $C$ \& LISANSKY, E. Alcohol and higher-order problem solving. Quarterly Journal of Studies on Alcohol, 1961, 22, 183-222.

CARPENTER, J., \& ROSS, B. Effect of alcohol on short term memory. Quarterly Journal of Studies on Alcohol, 1965, 26, 561-579.

FRANKENHAEUSER, M., MYRSTEN, A. \& JARPE, G. Effects of a moderate dose of alcohol on intellectual functions. Psychopharmacologia, 1962, 3, 344-351. GOODWIN, D. W., POWELL, B., BREMER, D., HOINE, H., \& STERN, J. Alcohol and recall: State-dependent effects in man. Science, 1969, 163, 1358-1360.

LEWIS, E., DUSTMAN, R \& \& BECK, E. The effect of alcohol on sensory phenomena and cognitive and motor tasks. Quarterly Journal of Studies on Alcohol, 1969, 30, 618-633.

RUIZ, R. A., \& KRAUSS, H. H. Test-retest reliability and practice effect with the
Shipley-Institute of Living Scale. Psychological Reports, 1967, 20, 1085-1086.

SCHALOCK, R. L., \& WAHLER, H. J. Changes in Shipley-Hartford scores with five repeated test administrations: Statistical conventions vs behavioral evidence. Psychological Reports, 1968, 22, 243-246.

STORM, T \& CAIRD, W, K. The effects of alcohol on serial verbal learning in chronic alcoholics. Psychonomic Science, $1967,9,43-44$. 